\title{
Spike-timing-dependent plasticity steers new Parkinson-stimulation protocols
}

\author{
Marcel AJ Lourens ${ }^{1,2^{*}}$, Jasmine A Nirody ${ }^{3}$, Hil GE Meijer ${ }^{2}$, Lo J Bour ${ }^{1}$, Stephan A van Gils ${ }^{2}$ \\ From Twenty Second Annual Computational Neuroscience Meeting: CNS*2013 \\ Paris, France. 13-18 July 2013
}

\begin{abstract}
Introduction
In advanced Parkinson's disease (PD), deep brain stimulation (DBS) can be used to disrupt pathological activity in the basal ganglia, thereby reducing PD motor symptoms. The standard protocol for DBS, continuous high frequency stimulation of target cells, is applied notably in subthalamic nucleus (STN) or globus pallidus pars interna. It is proposed that short-duration desynchronizing stimulation protocols may also disrupt pathological synchronous activity [1]. Synaptic plasticity is supposed to be the underlying mechanism. The goal of this study is to explore, with a biophysically plausible model, the role of synaptic plasticity in stabilizing firing patterns in the basal ganglia. Moreover, we investigate how STN stimulation should be applied, such that it exploits synaptic plasticity most effectively to bring the network in a less synchronous state.
\end{abstract}

\section{Methods}

We use an existing STN-GPe network model consisting of 16 STN and globus pallidus pars externa (GPe) cells mutually connected via a sparse structured architecture [2]. The dynamics of each cell is described by a singlecompartment conductance-based model. We augment the model with a rule for spike-timing-dependent plasticity (STDP) for the inhibitory connections within GPe. The synaptic weight is updated with an additive nearestspike pair-based STDP rule. We apply DBS as a train of positive current pulses $(P(t))$ injected directly into each STN cell. $P(t)$ is administered according to two different protocols: 1) Standard DBS, i.e. continuous stimulation, in which all STN cells receive exactly the same stimulation signal; 2) Coordinated Reset (CR) stimulation, in

\footnotetext{
* Correspondence: M.A.J.Lourens@utwente.nl

${ }^{1}$ Academic Medical Center, University of Amsterdam, Amsterdam, 1105 AZ, The Netherlands

Full list of author information is available at the end of the article
}

which the STN population is subdivided in 4 groups. Each STN group receives its own $P(t)$ which is periodically on and off.

\section{Results and discussion}

An STDP rule that down-/up-regulates the synaptic weights between GPe cells when they fire in synchronized/uncorrelated manner, stabilizes network states. Both a healthy state with desynchronized dynamics and a PD state with synchronized dynamics stably coexist. Depending on the original firing pattern, the network with STDP is 'learning' either pathological or healthy dynamics by adapting its pattern of the synaptic couplings between the GPe cells. Since DBS can change the firing pattern of the stimulated cells, it can be used to 'teach' the network with STDP to display more healthy activity. Our results suggest that when a travelling wave short-duration desynchronizing stimulation is applied sufficiently long and with sufficiently high amplitude, it may profit from STDP to train the network to fire in a less pathological manner. In contrast, STDP has a negative effect when continuous stimulation is employed, in the sense that the network becomes more synchronized when stimulation is switched off. Since with this kind of stimulation most of the time DBS is turned off, it saves battery power and it leads to fewer negative side effects of DBS in comparison to the traditional continuous high frequency stimulation.

\footnotetext{
Acknowledgements

Financial support from the Dutch Organization for Scientific Research (NWO), on grant 635.100.019, From spiking neurons to brain waves.

\section{Author details}

${ }^{1}$ Academic Medical Center, University of Amsterdam, Amsterdam, 1105 AZ, The Netherlands. ${ }^{2}$ Department of Applied Mathematics, University of Twente, Enschede, 7500 AE, The Netherlands. ${ }^{3}$ Biophysics Graduate Group, University of California, Berkeley, Berkeley, CA 94720, USA.
}

Published: 8 July 2013 


\section{References}

1. Hauptmann C, Tass PA: Cumulative and after-effects of short and weak coordinated reset stimulation: a modeling study. J Neural Eng 2009, 6:016004.

2. Terman $\mathrm{D}$, Rubin JE, Yew AC, Wilson CJ: Activity patterns in a model for the subthalamopallidal network of the basal ganglia. J Neurosci 2002, 22(7):2963-2976

doi:10.1186/1471-2202-14-S1-P401

Cite this article as: Lourens et al.: Spike-timing-dependent plasticity steers new Parkinson-stimulation protocols. BMC Neuroscience 201314 (Suppl 1):P401.

Submit your next manuscript to BioMed Central and take full advantage of:

- Convenient online submission

- Thorough peer review

- No space constraints or color figure charges

- Immediate publication on acceptance

- Inclusion in PubMed, CAS, Scopus and Google Scholar

- Research which is freely available for redistribution

Submit your manuscript at www.biomedcentral.com/submit
Ciomed Central 